\title{
Las playlists como espacios de mediación y su concentración en Argentina
}

\section{The playlists as spaces for mediation and their concentration in Argentina}

\author{
Nahuel Marcelo Olguin \\ Universidad Nacional de Quilmes, Argentina. Contacto: nahuelmolguin@gmail.com
}

ARTÍCULO

Recibido: agosto de 2020

Aceptado: octubre de 2020

\section{Resumen}

Con la consolidación de internet y la exponencial multiplicidad de la oferta musical, las listas de reproducción representaron una herramienta útil para que los usuarios tuvieran una mejor y más personalizada experiencia dentro de las plataformas de streaming de música. Sin embargo, las playlists también se consagraron como nuevos espacios de mediación en donde los diferentes actores de la industria fonográfica intentan intervenir con el fin de obtener el mayor beneficio. Lo cual adquiere una trascendencia aún superior si se tiene en cuenta que el sector de la música grabada está excesivamente concentrado.

Este marco obliga a pensar cómo se conforman las listas de reproducción, hasta dónde llega su importancia en la industria fonográfica, quiénes son sus principales propietarios y qué implicancias puede generar un alto grado de concentración.

Palabras claves: playlists, concentración, mediación, spotify, industria musical

\begin{abstract}
With the consolidation of the internet and the exponential multiplicity of the musical offer, playlists represented a useful tool for users to have a better and more personalized experience within music streaming platforms. However, playlists were also established as new mediation spaces where the different actors of the phonographic industry try to intervene in order to obtain the greatest benefit. Which takes on an even greater significance when one takes into account that the music sector is excessively concentrated.

This framework forces us to think about how playlists are made up, how important they are in the phonographic industry, who their main owners are and what implications a high degree of concentration can generate.
\end{abstract}

Keywords: playlists, concentration, mediation, spotify, music industry 


\section{Introducción}

El proceso de digitalización ${ }^{1}$ y la popularidad global de las plataformas de streaming ${ }^{2}$ se han convertido en pilares fundamentales de la industria musical. Conforme con el Global Music Report 2019 de la International Federation of the Phonographic Industry (2020), los ingresos provenientes del streaming musical representaron, por primera vez en la historia, más de la mitad $(56,1 \%)$ de los ingresos de la industria fonográfica. Pero, por sobretodo, han despertado numerosas expectativas de democratización para la producción, distribución, comercialización y consumo.

Sin embargo, el escenario actual parece "dificultar un aprovechamiento de las nuevas tecnologías por parte de los actores de menor escala (músicos autogestionados, sellos independientes, distribuidoras locales, etcétera) que permita dar batalla a los altos índices de concentración que históricamente caracterizaron al sector" (Moreno y Quiña, 2018, p. 2). En cambio, pese a las afirmaciones que atraviesan tanto a músicos como a agentes del negocio digital e incluso a las políticas públicas vinculadas, esto no supone una verdadera revolución, una ruptura brusca con la historia anterior, sino una línea de continuidad necesariamente contextualizada y determinada por las grandes transformaciones experimentadas por la cultura industrializada, especialmente en los años 80 y 90 como son la desregulación, la concentración y globalización e incluso la financiarización de la cultura (Lacroix y Tremblay, 1995).

Las nuevas tecnologías no pueden borrar la naturaleza central de las industrias culturales en la sociedad capitalista y los sectores más concentrados son quienes acaparan las ventajas del cambio digital en virtud de su gran tamaño y escala planetaria.

Desde finales de los años noventa, y sobre todo a comienzos del siglo XXI, las principales características socio-económicas de la industria fonográfica han propiciado procesos de concentración y transnacionalización. Solo tres compañías han sido protagonistas de una serie de compras y fusiones que delimitaron la repartición del mercado y las tendencias musicales. Según Nielsen (2013), Universal Music Group, Sony Music Entertainment y Warner Music Group controlan el $88.5 \%$ del mercado global.

Con el surgimiento de nuevos actores del sector tecnológico como Spotify, Apple Music, Deezer y Youtube, y la posibilidad de escuchar enormes catálogos de música dónde, cuándo y cómo el usuario quiere, parecía que el escenario cambiaría. Sin embargo, lejos de desconcentrarse, la industria fotográfica continuó su rumbo y, a su vez, encontró nuevos espacios de mediación: las listas de reproducción.

Actualmente, los discos constituyen el $22 \%$ del tiempo total de escucha, mientras que las playlists representan un 31\% del mismo (Music Business Association, 2016). A su vez, las listas de reproducción se han convertido en una importante herramienta de difusión para que los

\footnotetext{
${ }^{1}$ La digitalización se refiere al movimiento que permite el almacenamiento, reproducción y transmisión de piezas de contenido de los medios en la forma de datos binarios consistentes en ceros y unos (Doyle, 2002).

${ }^{2}$ Que permiten consumir bienes culturales a través de Internet y sin necesidad de descarga.
} 
usuarios descubran nuevas canciones y artistas. Con solo un click, las personas pueden escuchar lo que equivale a su propia emisora personalizada.

Teniendo en cuenta esto, el presente artículo se centrará en las acciones de la sueca Spotify, pionera de la música en streaming desde 2006, con el objetivo analizar el papel que juegan las playlists como espacios de mediación en el modelo de las industria fonográfica y cuál es su grado de concentración de las 50 listas de reproducción más populares de Argentina. Para ello, será necesario conocer las características de estas listas, saber cómo se conforman, quiénes son sus principales propietarios e impulsores y cuáles pueden ser las consecuencias de una excesiva concentración de estos espacios.

Si bien no se le puede adjudicar creación de las playlist a Spotify, lo que diferencia a esta plataforma es el foco puesto en la creación de un modelo de negocios rentable que, según datos oficiales, en julio de 2020 se consolidó como la plataforma líder en el mercado global con 299 millones de usuarios activos (el doble que Apple Music, su principal competidora) y presencia en 92 mercados del mundo (Spotify, 2020).

\section{Metodología}

En cuanto a la metodología aplicada, la presente investigación es de carácter descriptiva y se propone examinar características y funciones de las listas de reproducción de Spotify, como también su importancia para la industria de la música grabada y su nivel de concentración.

El trabajo se llevó a cabo a través de un análisis de tipo cuantitativo y cualitativo que articuló la revisión y sistematización de las referencias bibliográficas disponibles, la recolección de datos, la evaluación de los mismos y la confección de tablas y gráficos para su presentación.

En virtud de ello, fue necesario clasificar las listas de reproducción a partir de cómo se conforman. Es decir, de qué forma y quiénes realizan la curaduría de las canciones que componen las playlists. De este modo, se distinguen tres grandes grupos, las listas generadas por algoritmos, las que son gestionadas por los equipos de trabajo de Spotify y aquellas creadas por los propios usuarios de la plataforma

En cuanto a la indagación acerca de los niveles de concentración de las playlists, se tomaron como muestra las 50 listas de Spotify con mayor cantidad de seguidores en Argentina durante la primera semana de septiembre del 2020. A partir de allí, se recolectó y examinó los nombres de listas, a quién o quiénes pertenecen las mismas y el número de seguidores que ostentan cada una. Estos datos fueron recogidos íntegramente del sitio web especializado Charmetric.com. Posteriormente, se procedió a calcular el grado de concentración de propiedad y de las audiencias dentro de la muestra relevada.

Cabe aclarar en pos de la transparencia de este artículo que la información que exhibe Charmetric.com refiere a la totalidad de seguidores por lista y no distingue a los mismos por nacionalidad. En otras palabras, si bien Baila Reggaeton es la playlist con mayor audiencia en 
Argentina, los 10.116.038 de usuarios que la siguen no son necesariamente argentinos. Por lo tanto, los porcentajes de concentración no son absolutamente precisos, pero colaboran para ilustrar la situación actual en un primer acercamiento a los datos.

\section{Marco teórico}

Este trabajo se enmarca en las ideas de la Economía Política de la Comunicación que resaltan la necesidad de estudiar las dinámicas reales subyacentes al universo de las industrias culturales, que son reguladas estable y progresivamente por la lógica de la producción global de mercancías. Según esta perspectiva, es de suma importancia conocer las lógicas económicas que están insertas en la producción cultural industrializada, para comprender los procesos de difusión de bienes simbólicos. Pero al hacerlo, no se debe caer en el reduccionismo económico. Como argumenta Vincent Mosco, la Economía Política de la Comunicación puede ser entendida como una puerta de entrada a la comprensión de los fenómenos comunicacionales, pero de ninguna manera puede aspirar a dar cuenta de forma completa del fenómeno comunicacional (Mosco, 2006). Es claro que la economía no es el único factor en juego, pero no se la puede ignorar (Murdock y Golding, 1981), ya que es condicionante del proceso productivo.

En ese sentido, podemos establecer que la Economía Política de la Comunicación ha centrado sus líneas de estudio en dos grandes áreas. La primera, en la búsqueda de la naturaleza económica de los medios de comunicación, las industrias culturales y los sistemas comunicativos, y su relación con la estructura social más amplia. Y la segunda, en la observación específica de cómo la propiedad, los mecanismos de financiamiento y las políticas públicas influyen en los contenidos y el comportamiento de los medios (McChesney, R, 2000).

Para este trabajo usaremos el concepto de industrias culturales que Ramón Zallo (1988) definió como:

Un conjunto de ramas, segmentos y actividades auxiliares industriales productoras y distribuidoras de mercancías con contenidos simbólicos, concebidas por un trabajo creativo, organizadas por un capital que se valoriza y destinadas finalmente a los mercados de consumo con una función de reproducción ideológica y social- (p. 26).

Además, Zallo se dedicó a trabajar con la especificidad de las industria culturales, es decir, en qué aspectos se diferencian de otras industrias o áreas de la producción. Para ello, el académico enumeró ciertas características económicas de la cultura, entre las que se destacan, su alta aleatoriedad de la demanda, que implica un trabajo creativo, que supone una constante necesidad de renovación y que son bienes de oferta múltiple. Para este artículo nos interesa este último rasgo de la cultura, ya que implica que en cada individuo puede haber un creador y que el nivel de producción es mucho mayor que la oferta que es presentada al mercado, dos cualidades que se van profundizar con la aparición de internet. 
En los de comienzos del siglo XXI, la desmaterialización de la música a través de su formato mp3, la aparición de softwares de distribución de música gratuita como Napster y la posibilidad de un contacto más directo entre artistas y fans, llevaron a muchos teóricos a vaticinar una revolución rupturista que acabaría con la industria de la música grabada. Sin embargo, ya en 1998, Lawrence Lessig advertía que lejos de esa cierta imagen falsa del ciberespacio como espacio inherentemente en donde los usuarios podrían transitar libres del control, este tenía (y sigue conservando) el potencial de ser el espacio más plena y extensamente regulado que hayamos conocido jamás. Para Lessig, el entorno digital está atravesado por cuatro tipo de restricciones de la conducta que operan conjuntamente y la ley es solo una de ellas. Además, están las normas sociales, el mercado y, la más importante, la cual Lessig (1998) denominó como arquitectura o código y que consiste en:

El conjunto de protocolos y reglas implementadas, o codificadas, en el software del ciberespacio mismo, las cuales determinan cómo interactúan, o existen, las personas en este espacio. Este código, al igual que la arquitectura en el espacio real, establece los términos en los que entro, o existo, en el ciberespacio. $Y$ al igual que la arquitectura, no es opcional. No elijo si obedezco las estructuras que establece el código (p. 4).

Con el crecimiento económico, politico y simbólico de internet, las afirmaciones de Lessig toman mayor relevancia. Nick Srnicek (2018) sostiene que somos contemporáneos a una economía de plataformas y define a estas como infraestructuras digitales que permiten que dos o más grupos interactúen. Para Srnicek (2018), "las plataformas se posicionan como intermediarias que reúnen a diferentes usuarios: clientes, anunciantes, proveedores de servicios, productores, distribuidores e inclusos objetos físicos" (p. 45).

De esta manera, el principal atractivo o valor agregado de plataformas digitales de streaming musical, como Spotify o Apple Music, es ayudar a las personas a descubrir la música que les gusta, a los anunciantes a encontrar a posibles clientes y a los músicos a conseguir nuevos fans, todo por medio de las playlists y los algoritmos. Es decir, ante la exponencial multiplicidad de la oferta (actualmente Spotify posee en su catálogo 35 millones de canciones), los nuevos intermediarios generaron barreras en el espacio que ellos administran, a partir del código (la arquitectura de la home, los motores de búsqueda, los algoritmos, etc.), con el argumento de socorrer a los diferentes usuarios a tener una mejor experiencia y se posicionaron como "mediadores culturales" (Zallo, 2011, p. 163) que ordenan las relaciones en la oferta y ejercen de decisores de tendencias.

Así pues, las plataformas admiten de manera tácita su control y los usuarios son "libres" de aceptar esas condiciones o no, dependiendo de si desean acceder al servicio que estas aplicaciones proporcionan. A su vez, "casi siempre, estas plataformas vienen con una serie de herramientas que permiten a los usuarios construir sus propios productos, servicios y espacios de transacciones" (Srnicek, 2018, p. 45). En el caso de los servicios de reproducción de música por streaming, estos instrumentos son las listas de reproducción. De este modo, las plataformas 
aprovechan que cada usuario pueda generar y administrar sus propias playlists, para alimentar nuevamente esa falsa creencia que Lessig advirtió, porque plantean a las listas de reproducción como espacios libres de control, totalmente democratizadas y en donde, si se hacen las cosas bien, cualquier músico puede construir su propio éxito (Spotify, 2018a).

\section{Las playlist y cómo se conforman}

Podemos distinguir las listas de reproducción en tres tipos diferentes a partir de cómo se conforman: Las algorítmicas, las editoriales y las creadas por los usuarios.

Las playlist algorítmicas son únicas para cada oyente y se desarrollan a partir de los gustos específicos de cada persona. Estas playlist se basan en lo que comúnmente se conoce como crowdsourcing o colaboración abierta distribuida y consiste en externalizar tareas que, tradicionalmente, realizaban empleados o contratistas, dejándolas a cargo de un grupo numeroso de personas o de una comunidad. Es decir, los algoritmos observan de cerca los diferentes hábitos de escucha de los usuarios (qué les gusta, qué comparten y cuáles son las canciones que omiten) y los de aquellos con gustos similares, para luego predecir qué podrían querer escuchar a continuación. De esta manera, el ciclo de la lista de reproducción nunca se detiene.

Entre las playlists algorítmicas más populares se encuentran los Daily Mix, Discover Weekly, Release Radar, On Repeat y Repeat Rewind. Además, de los Top Charts que cada semana muestran las canciones más escuchadas y los Spotify Viral que contempla no sólo el volumen de escuchas, sino que además toma en cuenta el número de veces que se ha compartido una determinada canción.

Por su parte, las listas editoriales son aquellas que están curadas por los equipos de trabajo de Spotify. Estos especialistas se encargan de estudiar las estadísticas de los oyentes, para entender qué canciones se adaptan mejor a qué listas de reproducción. Algunas de las listas más populares son Rap Caviar, ¡Viva Latino! o Are \& Be. La elección de las canciones se basa en el gusto de los usuarios y las tendencias populares que puedan surgir de aplicaciones como Shazam, Tik Tok, blogs y revistas o cualquier otra plataforma online. Siguiendo a Lee Parsons, CEO de Ditto Music, en su libro Playlisting (2018):

Spotify trabaja con un sistema de niveles o modelo pirámide, similar al formato de la radio convencional. Los artistas más grandes van directamente al nivel más alto basado en popularidad. Los artistas nuevos pueden aparecer en las playlist virales, nuevos descubrimientos y luego (basados en la reacción de los usuarios) ser trasladados a cientos de diferentes playlists (p. 10).

Asimismo, dentro de las listas de reproducción editoriales, existe una subcategoría de playlists editoriales "personalizadas". Combinando el trabajo de los editores de Spotify con la tecnología de los algoritmos y el machine learning. El objetivo de esta fusión es generar una experiencia única para cada usuario a partir de sus reproducciones y búsquedas previas. 
Sin embargo, del total de 7.489.112 listas de reproducción que actualmente están disponibles en la plataforma, la gran mayoría de las playlists son creadas por los usuarios a partir de su propio criterio. Cualquier persona registrada en Spotify puede generar y compartir listas de reproducción. Las listas pueden ser públicas o privadas y se conforman a partir de las canciones, los artistas o los discos favoritos de los oyentes e incluso algunas se desarrollan para escuchar durante una actividad en particular como una fiesta o un viaje en auto. Además, Spotify permite que se puedan crear listas de manera colaborativa entre varios usuarios.

Esta herramienta no solo es utilizada cotidianamente por las personas y/o grupos de personas, las entidades económicas y empresas también aprovechan las ventajas de crear y controlar sus propias playlists. Por ejemplo, la fábrica surcoreana de autos Kia, la bebida isotónica Gatorade y también los grandes sellos discográficos como Warner, Universal y Sony, que administran las listas a través de firmas que han adquirido como Topsify, Digster y Filtr respectivamente.

\section{La importancia de las playlist}

Apreciar la importancia de las listas de reproducción es comprender la historia reciente de las tendencias en la música digital.

A comienzos de los años 2000, la descarga de canciones de manera individual fue un éxito inmediato. Con la digitalización y la aparición de dispositivos como los IPod y los MP3, la música dejó de estar atada a un soporte físico. Cualquier persona podía obtener, legal o ilegalmente, una sola canción a la vez. De esta manera, la reproducción tampoco quedó sujeta a los límites de la unidad física del disco y aparecieron las primeras listas.

Hoy en día, las descargas quedaron en el pasado. Las plataformas de streaming permiten a los usuarios acceder a enormes bibliotecas de música de forma online y se terminaron los problemas del espacio de almacenamiento. Las playlist de estos servicios tomaron la posta donde las listas de reproducción de los MP3 la habían dejado e hicieron de estas una de sus principales funciones de valor agregado. Conforme con el reporte U.S. Music 3602017 (Nielsen, 2017), casi tres cuartos $(74 \%)$ de los encuestados aseguraron que escuchan música en streaming a través de playlist, mientras que más de un tercio (38\%) admitió que las listas de reproducción representan una parte importante de su experiencia en estas plataformas. Esto toma aún más relevancia, teniendo en cuenta que, de acuerdo con el Global Music Report de la International Federation of the Phonographic Industry (2020) la suscripciones de pago a servicios de streaming volvieron a ser un factor impulsor clave (crecieron $33,5 \%$ ) de la industria de la música y que casi todos los mercados tuvieron crecimiento en esa área.

Las playlists también se transformaron en un factor clave a la hora de hallar música, ya que funcionan como guía para los usuarios que navegan entre los enormes catálogos de las plataformas de streaming. Actualmente, los usuarios de Spotify descubren en promedio 26 artistas 
nuevos al mes (Navas Rosal, 2016), algo impensado hasta hace algunos años. Entonces, donde los sellos discográficos alguna vez se desvivieron para que sus músicos sonarán en las radios y se convirtieran en portada de las revistas de música, hoy el objetivo es que las canciones lleguen a las principales listas de plataformas como Spotify o Apple Music. De acuerdo con Stuart Bell, cofundador de Dawbell, una compañía de relaciones públicas que representa a figuras como Rihanna, Paul McCartney, Pharrell Williams y Elton John, entrar en las listas de reproducción más grandes de Spotify es vital y el equivalente, hace diez años, a ingresar en las listas de hits de las principales radios del mundo (lqbal, 2019). Dos de las mayores historias de éxito de las listas de reproducción de Spotify, hasta el momento, son Lorde, quien apareció en la playlist Hipster International del cofundador de Napster, Sean Parker, antes de convertirse en una estrella internacional y Hozier, que en 2014 obtuvo el $46 \%$ de sus reproducciones gracias a las listas editoriales de la plataforma (Hogan, 2015). Pero, según denunció la Association of Independent Music (AIM), esas oportunidades son pocas y lejanas para el sector independiente (Jones, 2016). Parece necesario examinar quiénes son los propietarios de las playlists más populares y cuál es el grado de concentración de las audiencias para dar cuenta de las presiones y determinaciones que se dan dentro de estos espacios.

Finalmente, gracias a las plataformas digitales y sus estrategias basadas en el análisis de grandes bancos de datos, las listas de reproducción le abrieron la puerta a un nuevo paradigma en el que las personas experimentan y descubren la música. Los responsables de Spotify entendieron que las playlists posibilitaron nuevos tipos de empaquetamiento de la música. A las listas de reproducción basadas en el contenido (género, idioma, zonas geográficas, etc.) que ya conocíamos, se le sumaron nuevas listas basadas en el contexto (Joven y Jenkins, 2018) que están pensadas para disfrutar actividades, eventos o momentos. De esta manera, surgen listas de reproducción diseñadas exclusivamente para, por ejemplo, salir a correr, una tarde de lluvia, una cena romántica o un domingo a la mañana. En 2015, el CEO y fundador de Spotify, Daniel Ek, aseguró que para que Spotify sea la banda sonora de nuestra vida, es necesario poder ofrecer música basada en quiénes somos, qué estamos haciendo y cómo nos sentimos momento a momento (Segall, 2015). Si bien, las playlists basadas en el contenido siguen siendo la forma en que la mayoría de los usuarios están acostumbrados a navegar, más de un tercio comenzó a elegir las listas basadas en el contexto (Joven y Jenkins, 2018). Si este cambio en el hábito de consumo sigue creciendo, puede significar nuevos desplazamientos dentro de la industria en un futuro próximo (Forde, 2017).

\section{La concentración de las listas}

Conforme con el sitio especializado Spot on Track, Spotify cuenta con 7.489 .112 listas de reproducción en todo el mundo en septiembre de 2020. La compañía sueca es propietaria y gestiona tan solo $55.482(0.74 \%)$ de estas playlist. Mientras que los usuarios aprovechan el 
espacio semiestructurado de la plataforma para tener un rol activo en donde proponen sus propias curadurías en base a sus gustos y necesidades. El informe U.S. Music 3602017 (Nielsen 2017) confirmó que el $58 \%$ de las personas crean sus listas de reproducción y el $32 \%$ comparte sus playlist con otros usuarios.

No obstante, Luis Aguiar y Joel Waldfoge en su trabajo Platforms, Promotion, and Product Discovery (2018) fueron los primeros en hacer referencia de los altos niveles de concentración de las audiencias que se expresan en las playlist de Spotify. En su análisis, los investigadores examinaron las 1.000 listas de reproducción con mayor número de seguidores y descubrieron que las playlists que son propiedad de Spotify concentran más de tres cuartas partes del total del público. Le siguen en porcentaje de seguidores acumulados las listas gestionadas por los principales sellos discográficos (Filtr, Digster y Topsify). Por su parte, los propietarios restantes de la lista de reproducción (sellos independientes, medios de comunicación, pequeñas empresas dedicadas a la creación y gestión de playlists, marcas y usuarios) tienen participaciones insignificantes.

En Argentina, cuando se observa a los propietarios de las 50 playlists con mayor cantidad de seguidores, el panorama es similar aunque presenta algunas diferencias. En primer lugar, el predominio de Spotify es ineludible e incluso superior que a nivel global. La plataforma gestiona 18 de las 25 listas de reproducción más populares en el país y concentra el $92.5 \%$ de la audiencia. Por su parte, 1.117 .425 de los usuarios (4.8\%) eligen aquellas listas que pertenecen a usuarios ajenos a la industria fonográfica que, lejos de tener participaciones poco significantes, comprenden el segundo porcentaje más alto de seguidores en Argentina. En tercer y cuarto puesto se encuentran Warner Music Group (1.4\%) y Sony Music (0.8\%) respectivamente y, de acuerdo con el relevamiento, Universal Music Group no posee ninguna playlist dentro de las 50 listas de reproducción más populares. Por último, dos sellos independientes nacionales como Mueva Records (65.562) y Magenta Discos (52.597) concentran el 0.5\% de las escuchas.

\begin{tabular}{|r|l|l|r|}
\hline \multicolumn{4}{|c|}{ Resultados de Chartmetric sobre las 50 playlist con más seguidores en Argentina } \\
(Del 1 al 8 de septiembre de 2020)
\end{tabular}




\begin{tabular}{|c|c|c|c|}
\hline 9 & Vamo Lo Pibe & Spotify & 380.256 \\
\hline 10 & Los 90 Argentina & Spotify & 249.362 \\
\hline 11 & Los 2000 Argentina & Spotify & 238.812 \\
\hline 12 & Pop Argentina & Spotify & 226.997 \\
\hline 13 & Los 80 Argentina & Spotify & 207.523 \\
\hline 14 & Rock nacional & cafedelaplazasn & 171.224 \\
\hline 15 & Volvieron los Lentos & Topsify Argentina & 152.470 \\
\hline 16 & Previa Latina en CASA & Topsify Argentina & 151.982 \\
\hline 17 & Previa y cachente - Fer Palacio & ohhmykastek & 149.278 \\
\hline 18 & This is Charly Garcia & Spotify & 127.694 \\
\hline 19 & Argentina Viral 50 & spotifycharts & 126.176 \\
\hline 20 & This is: Rodrigo & Spotify & 118.726 \\
\hline 21 & Folklore de Siempre & Spotify & 94.276 \\
\hline 22 & Los Redondos - Indio Solari & Uriel Koffman & 92.190 \\
\hline 23 & Cumbia del recuerdo & landri_04 & 91.904 \\
\hline 24 & This is Ciro & Spotify & 85.242 \\
\hline 25 & HITS EN INGLÉS 2020 -Hits Para NO Parar & Filtr Argentina & 80.160 \\
\hline 26 & Cachengue 2020 & Pedro Espinal & 75.693 \\
\hline 27 & Previa - Flanchota & Flanchotaok & 72.125 \\
\hline 28 & This is: Gilda & Spotify & 71.200 \\
\hline 29 & La Previa Argentina & Maxi Jayat & 68.430 \\
\hline 30 & Suena Fresco & Mueva Records & 65.562 \\
\hline 31 & FIESTA x ZOOM 2020 CON Tommy Muñoz & Filtr Argentina & 65.263 \\
\hline 32 & Trap Argentino - Trap Argentina & Matty Yarza & 64.280 \\
\hline 33 & Cuarteto y cumbia clasicos & 2137y4jcuguuvpld4ol7e6ahy & 63.979 \\
\hline 34 & $\begin{array}{l}\text { TOP } 50 \text { ARGENTINA } \square \square \text { TOP } 50 \text { ÉXITOS } \\
2020\end{array}$ & pcnaimad & 61.705 \\
\hline 35 & Lentos Latinos & Carolina Soria & 55.321 \\
\hline 36 & Ulises Bueno & discosmagenta & 52.597 \\
\hline 37 & This is: Kevin Johansen & Spotify & 50.793 \\
\hline 38 & No Te Va A Gustar & Wen Santacruz & 48.503 \\
\hline 39 & Rock Argentino & Ati Maldonado & 45.782 \\
\hline 40 & One 103.7 (Actualizada semanalmente) & Radio One 103.7 & 44.645 \\
\hline 41 & VERANO $2020 \square$ SUMMER SET 2020 By & Filtr Argentina & 40.920 \\
\hline
\end{tabular}




\begin{tabular}{|r|l|l|r|}
\cline { 2 - 3 } & Tommy Muñoz & & \\
\hline 42 & $\ldots$ & Marcos Fernandez Loyarte & 35.388 \\
\hline 43 & PREVIA CUMBIANCHA $\square$ & Felipe Vallesi & 35.212 \\
\hline 44 & Te Amo Tanto & Topsify Argentina & 29.829 \\
\hline 45 & This is: Onda Vaga & Spotify & 29.607 \\
\hline 46 & Boliche 2020 & Facu Fica & 24.924 \\
\hline 47 & Folklore Argentino 2020 & Top Music & 22.825 \\
\hline 48 & cumbia santafesina & Lucas Ciafardini & 22.756 \\
\hline 49 & Los redondos/La renga & Andie Lucchetti & 22.495 \\
\hline 50 & Aspen 102.3 & Fernando Pereira Arias & 20.869 \\
\hline
\end{tabular}

\section{Concentración de audiencia en las playlist de Spotify Argentina}

Datos relevados de las 50 playlist con más seguidores segun Charmetric (10 de septiembre 2020).
Spotify
Otros
Topsify
Filtr
Sellos independientes

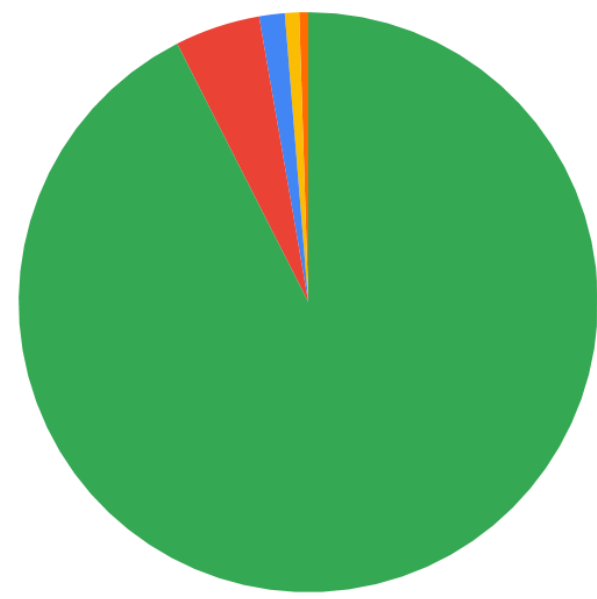

\section{Los riesgos de la concentración}

Los procesos de concentración son defendidos por algunos autores, que sostienen que proporciona una mayor estabilidad a los mercados. Sin embargo, Juan Carlos Miguel de Bustos (2003) considera que la concentración se transforma en una cuestión delicada cuando se aplica a cualquier sector de las industrias culturales, porque "sobrepasa las opciones de tipo económico para entrar de lleno en una multiplicidad de implicaciones" (p. 122).

La fuerte concentración de las audiencias en manos de Spotify tanto a nivel mundial como nacional, no solo plantea una continuación de las imperfecciones y asimetrías del paradigma de la industria musical previa al surgimiento del streaming, sino también una serie de posibles consecuencias que deben ser tenidas en cuenta: 
a) Una relativa homogeneización de la producción

Autores como Mosco (1996) o McChesney (2002) sostienen que la excesiva concentración en el ámbito de las industrias culturales supone una seria amenaza a la diversidad cultural. Esto se debe, al menos en parte, a que uno de los mayores desafíos dentro de las industrias culturales es la alta aleatoriedad de la demanda. Si la presencia en las listas más populares puede asegurar el éxito de reproducciones, es muy probable que los músicos comiencen, consciente o inconscientemente, a producir canciones pensando en alcanzar estas listas y sus enormes audiencias.

De este modo, si más del 90\% de las listas más escuchadas son administradas por Spotify, hay una gran probabilidad de que los criterios de selección musical de los grupos editoriales y los algoritmos de esta plataforma terminen atravesando el trabajo creativo de los artistas. Lo cual se profundiza aún más con herramientas como Spotify for Artists que estimulan a que los artistas se guíen cada vez más por los datos a la hora de crear sus obras.

Asimismo, los algoritmos no están diseñados para la sorpresa, sino para maximizar el disfrute y el tiempo dentro de estas plataformas, sugiriendo artistas o canciones similares a las que los usuarios escucharon con anterioridad. De esta manera, se crean cámaras de eco basadas en datos que estimulan a un tipo (o subgénero) en particular de música al que el crítico del The New York Times, Jon Caramanica, ha denominado Spotifycore ${ }^{3}$ en varias notas.

b) La perpetuación de la desigualdades

Al igual que en el punto anterior, el foco se mantiene en las cámaras de eco, propias de los algoritmos y el diseño de Spotify. En una entrevista para la National Public Radio (2018), Cathy O'Neil, autora del libro Armas de destrucción matemática, explicó que:

Los algoritmos no hacen las cosas justas. Repiten nuestras prácticas pasadas, nuestros patrones. Automatizan el status quo. Eso sería genial si tuviéramos un mundo perfecto, pero no lo tenemos (...) esto significa que podrían estar codificando el sexismo o cualquier otro tipo de intolerancia.

En consonancia con las afirmaciones de O'Neil, investigaciones recientes (Pelly, 2018) sobre el sesgo de género en las listas de reproducción más populares de Spotify revelaron que, en el transcurso de un mes, el $64,5 \%$ de las canciones presentes en Today's Top Hits pertenecían a cantantes hombres, un $20 \%$ a mujeres y un $15.5 \%$ a colaboraciones entre hombres y mujeres.

\section{c) La censura}

\footnotetext{
${ }^{3}$ Una mezcla de pop, con el ritmo del tambor lento del hip hop, el drop de la EDM (Electronic Dance Music) y la sensibilidad de la melodía del R\&B.
} 
En 2018, se desató una fuerte polémica a partir de la decisión de Spotify de retirar de sus playlist más populares a los raperos R. Kelly y XXXTentacion por una nueva política de mala conducta (Leight, 2018). Si bien, semanas más tarde, el servicio de streaming anuló esta decisión, el hecho por sí mismo alimentó las preocupaciones de censura.

La situación demostró que Spotify, en lugar de de ser una plataforma neutral, puede aprovechar su posición como administrador de las listas de reproducción con mayor cantidad de seguidores para comportarse como un goalkeeper y, prácticamente, desterrar a la marginalidad a ciertos artistas, si así lo deciden sus autoridades.

\section{Conclusiones}

A lo largo de este artículo, se ha expuesto que las listas de reproducción son un espacio de interacción que se conforma por la participación de los diferentes actores de la industria musical y que, además, están atravesadas por los cuatro tipos de restricción de la conducta que operan conjuntamente.

Comprender la relevancia de las playlists para el modelo actual de la industria de la música grabada y analizar los números acerca de su extremada concentración de la audiencia (al menos en el caso de Spotify), ayudan a entender que, a pesar de los enormes catálogos musicales presentes en los servicios de streaming, una gran cantidad de los usuarios se concentran en listas que son gestionadas por muy pocos actores. Con la digitalización la oferta de artistas, canciones y géneros es cada día es más diversa, pero los criterios de selección musical, dentro de estos espacios de mediación de la industria, están concentrados.

Tanto a nivel global como a nivel nacional, las nuevas tecnologías (en este caso, las listas de reproducción) no han derribado las lógicas y las asimetrías de un sector tan concentrado como lo es el de la música. Por el contrario, suponen una línea de continuidad en donde las nuevas plataformas digitales, como Spotify, se han apoderado de ellas para ocupar un lugar en la mesa chica.

De igual manera, parece sustancial recalcar que, como en el caso de otras industrias culturales, el consumo de música en playlists es un proceso constante y dinámico que no se acaba en la simple recepción. Más de la mitad de las personas crean sus listas de reproducción y un tercio de los usuarios las comparte. Por su parte, se ha verificado que los argentinos tienen un mayor interés por las playlists que pertenecen a propietarios ajenos a la industria fonográfica que el promedio global.

Si bien en este trabajo se han enumerado posibles consecuencias fruto del panorama actual en el mundo y la Argentina, se deberá prestar atención a las estrategias de Spotify en el futuro, teniendo en cuenta su trascendencia como nuevo mediador cultural. Fundamentalmente, si se considera la suspensión de su iniciativa que permitía a los artistas independientes subir su música directamente a la plataforma (Spotify, 2019), una jugada que dejaba afuera a los sellos 
discográficos y las distribuidoras digitales (similar a lo que Amazon está llevado adelante en la industria editorial con Amazon Kindle Direct Publishing), y su reciente giro de atención hacia el mundo de los podcasts (Valinsky, 2019).

\section{Referencias bibliográficas}

Aguiar, L. y Waldfogel, J. (2018). Platforms, Promotion, and Product Discovery: Evidence from Spotify Playlists. JRC Digital Economy Working Paper 2018-04; JRC Technical Reports, JRC112023.

Bustamante, E. (2003). Hacia un nuevo sistema mundial de comunicación : las industrias culturales en la era digital. Barcelona: Editorial Gedisa.

Doyle, G. (2002). Understanding media economics. Londres: SAGE Publications.

Forde, E (2017). They could destroy the album: how Spotify's playlists have changed music for ever. Recuperado de: https://www.theguardian.com/music/2017/aug/17/they-could-destroy-thealbum-how-spotify-playlists-have-changed-music-for-ever

Hogan, M. (2015). Up Next: How Playlists Are Curating the Future of Music. Recuperado de: https://pitchfork.com/features/article/9686-up-next-how-playlists-are-curating-the-future-of-music/ International Federation of the Phonographic Industry (2020). Global Music Report: the industry in 2019. Recuperado de: https://www.ifpi.org/wp-content/uploads/2020/07/Global_Music Reportthe Industry in 2019-en.pdf

Iqbal, N. (2019). Forget the DJs: Spotify playlists are the new musical starmakers. Recuperado de: https://www.theguardian.com/music/2019/apr/28/streaming-music-algorithms-spotify

Jones, R. (2016). Streaming playlists becoming 'closed shop' to independent labels. Recuperado de: $\quad$ https://www.musicbusinessworldwide.com/streaming-playlists-becoming-closed-shop-toindependent-labels/

Joven, J. y Jenkins, C. (2018). Spotify: The Rise of the Contextual Playlist. Recuperado de: https://blog.chartmetric.io/spotify-the-rise-of-the-contextual-playlist-c6f2c26900f4

Leight, E. (2018). Spotify Removes R. Kelly From Playlists, But Keeps Controversial Singer on Service. Recuperado de: https://www.rollingstone.com/music/music-news/spotify-removes-r-kellyfrom-playlists-but-keeps-controversial-singer-on-service-627732/

Lessig, L. (1998). Las leyes del ciberespacio, conferencia Taiwan Net '98, mimeo, Taipei. Recuperado de: http://www.uned.es/ntedu/espanol/master/segundo/modulos/audiencias-ynuevos-medios/ciberesp.htm

McChesney, R. W. (2000). The political economy of communication and the furure of the field. Londres: SAGE Publications.

McChesney, R. W. (2002). The Global Restructuring of Media Ownership. En Roboy, M. (Eds.), Global Media Policy in the New Millennium (pags 149-162). Lutton: Lutton University Press. 
Miguel de Bustos, J. C. (2003); Los grupos de comunicación: la hora de la convergencia. En Bustamante, E. (coord.), Hacia un nuevo sistema mundial de comunicación: las industrias culturales en la era digital (págs 227-256). Barcelona: Editorial Gedisa.

Mosco, V. (1996). Political Economy of Communication: Rethinking and Renewal. Londres: SAGE Publications.

Mosco, V. (2006). Economía Política de la Comunicación: una actualización diez años después. En Cuadernos de Información y Comunicación vol 11. Universidad Complutense de Madrid, p. 5779.

Music Business Association (2016). Playlist overtake albums in listenership, says loop study. Traducción propia. Recuperado de: https://musicbiz.org/news/playlists-overtake-albumslistenership-says-loop-study/

National Public Radio (2018). Cathy O'Neil: Do Algorithms Perpetuate Human Bias? Recuperado de: https://www.npr.org/transcripts/580617998?storyld=580617998? storyld=580617998

Navas Rosal, A (2016). Los usuarios de Spotify descubren en promedio 26 artistas nuevos al mes. Recuperado de: https://industriamusical.es/los-usuarios-de-spotify-descubren-en-promedio-26artistas-nuevos-al-mes

Nielsen (2013). The Nielsen Company \& Billboard's 2012 Music Industry Report. Recuperado de https://www.businesswire.com/news/home/20130104005149/en/Nielsen-Company-

Billboard\%E2\%80\%99s-2012-Music-Industry-Report

Nielsen (2017). U.S. Music 3602017 Report. Disponible en: https://www.nielsen.com/us/en/insights/report/2017/music-360-2017-highlights/

Parsons, L. (2018). Playlisting: The Billion Dollar Business. A complete guide to getting your music playlisted on Spotify, Apple Music and more. Amazon Digital Services LLC. Recuperado de: https://dashboard.dittomusic.com/playlisting-the-billion-dollar-business

Pelly, L. (2018). Sexism on Spotify. Recuperado de: https://thebaffler.com/latest/discover-weaklypelly

Segall, L. (2015). Spotify wants to be the soundtrack of your life de CNN Bussines (Traducción propia). Recuperdo de: https://money.cnn.com/2015/05/20/technology/spotifyannouncement/index.html

Spotify (2018a). The Stars of the Spotify Playlists. Recuperado de: https://artists.spotify.com/blog/the-stars-of-the-spotify-playlists

Spotify (2018b). The Top Songs, Artists, Playlists, and Podcasts of 2018. Disponible en: https://newsroom.spotify.com/2018-12-04/the-top-songs-artists-playlists-and-podcasts-of-2018/ Spotify (2019). We're Closing the Upload Beta Program. Here's What Artists Need to Know. Recuperado de: https://artists.spotify.com/blog/we\%27re-closing-the-upload-beta-program Spotify (2020). Spotify Reports Second Quarter 2020 Earnings. Recuperado de: https://newsroom.spotify.com/2020-07-29/spotify-reports-second-quarter-2020-earnings/ 
Srnicek, N. (2018). Capitalismo de plataformas, traducido por Caja Negra Editorial, Ciudad Autonoma de Buenos Aires.

Tremblay, G. y Lacroix, J. (1995). Les autoroutes de linformation. Un produit de la convergence. Québec: Presses de IUniversité du Québec.

Valinsky, J. (2019). Spotify bet on podcasts. It's working. Recuperado de: https://edition.cnn.com/2019/10/28/tech/spotify-third-quarter-earnings/index.html

Zallo, R. (1988). Economía de la comunicación y la cultura. Madrid: Akal.

Zallo, R. (2011). Estructuras de la Comunicación y de la Cultura. Políticas para la Era digital. Barcelona: Editorial Gedisa. 